\title{
PLANTAS ASSOCIADAS À VIDEIRAS: UMA ESTRATÉGIA PARA O CONTROLE BIOLÓGICO NO RIO GRANDE DO SUL
}

\author{
M. Diehl, N.J. Ferla, L. Johann \\ Centro Universitário UNIVATES, Laboratório de Acarologia, Rua Avelino Tallini, 171, CEP 95900-000, \\ Lajeado, RS, Brasil. E-mail:marciadiehl@universo.univates.br
}

\section{RESUMO}

Espécies de plantas associadas podem servir de abrigo para um grande número de organismo, entre eles ácaros predadores. Este estudo visou identificar as plantas associadas com maior potencial de inimigos naturais, para poder estabelecer formas de controle biológico. O estudo foi realizado nos municípios de Boqueirão do Leão e Dois Lajeados, no período de março de 2006 a fevereiro de 2007 e em Candiota e Bento Gonçalves, no período de outubro de 2006 a setembro de 2007, no Estado do Rio Grande do Sul. Os ácaros foram coletados mensalmente em cinco espécies de plantas comumente encontradas no interior ou nos arredores da cultura de videiras. As plantas foram avaliadas sob microscópio estereoscópico, sendo encontrado um total de 1.036 ácaros das famílias Phytoseiidae, Stigmaeidae e Iolinidae. Foram amostradas 80 espécies de plantas, totalizando 506 amostragens. Em Sonchus oleraceus L, foram encontrados 110 espécimes, em Plantago tomentosa Lam. foram encontrados 105 ácaros, em Chromolaena Laevigata (Lam) R. M. King \&H. Rob., 97 ácaros e em Solanum americanum Mill. 75 ácaros. Pronematus anconai Baker, (1943) 1944, foi mais abundante, com 618 espécimes presentes em 44 plantas amostradas, Transeius lisei Ferla \& Silva 2008, com 112 espécimes, Typhlodromalus aripo Deleon, 1967, com 50 espécimes e Arrenoseius gaucho Ferla et al. 2010, com 68 espécimes. As espécies de plantas associadas à videira apresentam um grande número de ácaros predadores, sendo que elas podem ser um reservatório de inimigos naturais para controlar ácaros praga nesta cultura. Desta forma, essas plantas podem ser utilizadas em estratégias de onde programas de controle biológico aplicado podem ser utilizados.

PALAVRAS-CHAVE:Inimigosnaturais, ácaros predadores, ácarosfitófagos, Phytoseiidae,Stigmaeidae.

\section{ABSTRACT}

PLANTS ASSOCIATED TO THE GRAPEVINE: A STRATEGY FOR BIOLOGICAL CONTROLINTHESTATEOF RIOGRANDEDOSUL, BRAZIL. Associated plant species can provide shelter for a large number of organisms, including predatory mites. This study aimed to identify the associated plants with the greatest potential for natural enemies, in order to establish forms of biological control. The study was conducted in Boqueirão do Leão and Dois Lajeados counties, from March 2006 to February 2007, and in Candiota and Bento Gonçalves counties, from October 2006 to September 2007, in the state of Rio Grande do Sul, Brazil. The mites were collected monthly on 5 plant species commonly found inside or outside the grapevine crop. The plants were evaluated under a stereoscopic microscope, and a total of 1036 mites of the Phytoseiidae, Stigmaeidae, and Iolinidae families were found. Eighty plant species were sampled, for a total of 506 samples. Examination revealed 110 specimens on Sonchus oleraceus L., 105 on Plantago tomentosa Lam., 97 on Chromolaena Laevigata (Lam) R. M. King \& H. Rob., and 75 on Solanum americanum Mill. Pronematus anconai, 1944, was the most abundant species, with 618 specimens present on 44 plants, followed by Transeius lisei, with 112 specimens, Typhlodromalus aripo Deleon, 1967, with 50 specimens, and Arrenoseius gaucho, Ferla et al. 2010 with 68 specimens. Plants associated to the grapevine have a large number of predatory mites, and they can be a reservoir of natural enemies for the control of pest mites in this crop. Thus, these plants can be used in strategies involving the use of biological control programs.

KEY WORDS: Natural enemies, predatory mites, phytophagous mites, Phytoseiidae, Stigmaeidae. INTRODUÇÃO 
Cultivos agrícolas são afetados por espécies de insetos eácaros que podem ocasionar sérios danos às plantas cultivadas no Brasil. Normalmente são controlados através da aplicação de produtos químicos, os quais provocam efeito negativo sobre o ambiente e ao próprio homem. Assim, surge a necessidade de conhecer novas formas de controle sem o uso desses produtos onde se destaca o controle biológico. Recentemente, aumentou a pressão para que o ambiente seja preservado e o uso de tecnologias limpas apresenta tendência de ser cada vez mais utilizado, juntamente com outras formas de controle que preservem o ambiente (PARRA et al., 2002).

O controle biológico constitui a regulação de populações de plantas e animais por inimigos naturais, que operam na mortalidade biótica (PARRA et al., 2002). Pode ser definido como a regulação da população de uma espécie dentro de certos limites, superior e inferior, por um período de tempo, por qualquer combinação de fatores naturais classificados como bióticos ou abióticos. Dentre os fatores bióticos, está a ação de inimigos naturais onde se destacam os predadores.

As plantas associadas, também conhecidas como plantas invasoras ou daninhas, ocorrem naturalmente e têm crescimento espontâneo em áreas de cultivo. Estudos mostram que a diversificação cuidadosa, adequada ou a simples manutenção de plantas associadas em agroecossistemas pode diminuir de maneira significativa as populações de espécies pragas (Altieri et al., 2003). Certas plantas associadas desempenham papel ecológico importante por hospedar e suportar um complexo de artrópodes benéficos que ajudam na supressão de pragas (Altieri et al., 1977).

Estruturas morfológicas como pelos, domácias, nectários, além de outras funções para a planta, oferecem abrigo e alimento a diferentes espécies de artrópodes predadores, dos quais muitos são eficazes no controle de diversas pragas (WALTER, 1996; AgRAWAL et al., 2000). Essas estruturas podem ser de grande importância para esses organismos, uma vez que podem favorecer sua manutenção na planta ao disponibilizarem alimento alternativo e refúgio contra seus próprios predadores (MATOs et al., 2006).

A presença de vegetação natural próxima a pomares beneficia a migração e manutenção deinimigos naturais no interior destes (Altieri et al., 2003; TIXIER et al., 2000). Plantas favoráveis para ácaros predadores fitoseídeos talvez possam ser utilizadas nas bordas para funcionar como refúgio e reservatório destes predadores (PRISChMANN; JAMES, 2003; MoraES et al., 2001).

O uso de ácaros predadores para o controle de ácaros pragas já vem sendo estudado e usado há bastante tempo (McMurtry; CROFT, 1997). Ácaros predadores são frequentemente encontrados associados a ácaros fitófagos na vegetação nativa, em culturas anuais e perenes. Entretanto, pouco se conhece a respeito da potencialidade de diferentes grupos de organismos atuarem como inimigos naturais de ácaros praga.

Os predadores mais promissores de ácaros fitófagos no campo pertencem às famílias Phytoseiidae, Stigmaeidae e Iolinidae (MCMURTRY et al., 1970; Moraes, 1991; Moraes et al., 2004).

Phytoseiidae tem recebido considerável atenção desde a década de 1950, quando se descobriu que algumas espécies eram importantes inimigos naturais de ácaros tetraniquídeos, tornando-os importantes nos programas de manejo integrado de pragas (MEYER, 2003). Estes ácaros são comumente encontrados nas partes aéreas das plantas, onde desempenham papel importante na manutenção de baixas populações de ácaros fitófagos (McMurTrY; CROFT, 1997). Na família Phytoseiidae, os ácaros generalistas se alimentam de pólen, fungos e ácaros e podem sobreviver em pomares em alta densidade mesmo quando as presas primárias são escassas ou temporariamente extintas. Enquanto que especialistas necessitam que uma determinada espécie de presa permaneça no ambiente, sendo que na sua ausência se extinguem localmente por falta de alimento (ALtieri, 2003; McMurtry; Croft, 1997; Moraes, 1991). Uma das características marcantes dos fitoseídeos como inimigo natural de ácaros praga é o seu ciclo de vida curto, quando submetidos a condições de temperatura e umidade favoráveis (MEYER, 2003).

Além dos fitoseídeos, outras famílias apresentam ácaros predadores de tetraniquídeos, destacando-se a família Stigmaeidae (LAING; KNOP, 1982), que apresenta menor diversidade e é representada principalmente por espécies dos gêneros Agistemus Summers, 1960 e Zetzellia Oudemans, 1927 (FerLa; Moraes, 2002).

A família Iolinidae tem sido referida por LAING; Knop (1982) e Perrin; McMurtry (1996) como predadora de Eriophyidae. Na Alemanha, Pronematus staerkiSchruft, 1972é um inimigo natural importante de Caliptrimerus vitis (Duso; LiLlo, 1996). Pronematus anconai foi observado, associado à cultura da videira no Rio Grande do Sul (JoHANN et al., 2009; KLOCK et al., 2011).

Considerando a importância das plantas associadas à videiras no Rio Grande do Sul, RS, torna-se necessário realizar estudos para identificar as espécies com maior potencial de inimigos naturais. Podendo assim estabelecer formas de controle biológico natural, com a manutenção e a preservação de inimigos naturais nestas áreas, mantendo as populações de ácaros fitófagos abaixo do nível de dano econômico.

Este trabalho objetiva identificar espécies de plantas associadas com maior potencial para representar reservatório de inimigos naturais de ácaros 
predadores na cultura da videira no Estado do Rio Grande do Sul.

\section{MATERIAL E MÉTODOS}

Este estudo foi conduzido em plantas associadas à vinhedos dos cultivares Cabernet Sauvignon e Bordeaux nos municípios de Dois Lajeados, posição: 22J 0414743, UTM: 6797765; Boqueirão do Leão, posição: 22J 0364927, UTM: 6752228 entre março de 2006 e fevereiro de 2007, e Bento Gonçalves posição: 22J 446541, UTM: 6767872 e Candiota posição: 22J 246629, UTM: 6515586, no período de outubro de 2006 a setembro de 2007 em cultivares Cabernet Sauvignon, Pinot Noir, Chardonnay e Merlot. Os quatro municípios estão localizados no RS.

Osácaros foram coletados mensalmenteem cinco plantas comumente encontradas no interior ou nos arredores da cultura de videira. As plantas coletadas foram individualizadas em sacos plásticos, guardados em caixa de isopor com Gelox®, para transporte ao laboratório onde as amostras foram processadas e identificadas com o uso de chaves dicotômicas e consulta a especialistas. Em cada um dos espécimes coletados foi realizado um esforço amostral de uma hora. A contagem foi realizada diretamente sobre as folhas, utilizando microscópio estereoscópico, observando as duas faces da folha. Com a utilização de pincel de ponta fina, os ácaros foram coletados e guardados em álcool $70 \%$ para posterior montagem e identificação. Todos os ácaros foram montados em meio de Hoyer (JEPPson et al., 1975). As lâminas montadas foram mantidas em estufa a $50-60^{\circ} \mathrm{C}$, por cerca de 10 dias para a fixação, distensão e clarificação dos espécimes e secagem do meio. A identificação dos ácaros foi feita com o auxílio de microscópio óptico com contraste de fase. Espécimes representantes de cada uma das espécies encontradas foram etiquetados e guardados em caixas de madeira, depositadas em ambiente climatizadoe depositadas na Coleção de Referência de Ácaros do Museu de Ciências Naturais do Centro Universitário UNIVATES (ZAUMCN), Lajeado, Rio Grande do Sul.

\section{RESULTADOS e DISCUSSÃO}

Foram amostradas 80 espécies de plantas, pertencentes a 23 famílias botânicas totalizando 506 amostragens, sendo que em 58 espécies foram encontrados ácaros predadores. Ao total, foram coletados 1.036 ácaros predadores, pertencentes a 35 espécies das famílias Phytoseiidae, Stigmaeidae e Iolinidae.

Asteraceae apresentou maior número de espécies amostradas, com 29 espécies (Tabela 1). Plantago tomentosa foi amostrada 40 vezes, sendo que em 38 amostras foram encontrados 105 ácaros de 17 espécies. Sonchus oleraceus foi amostrada 28 vezes, sendo que em 11 coletas foram encontrados ácaros. Nesta espécie vegetal foram observados 110 espécimes pertencentes a duas espécies acarinas, Neoseiulus californicus e Pronematus anconai. Chromolaena Laevigata amostrada apenas quatro vezes e nas quatro vezes encontrados 97 ácaros de quatro espécies. Em Solanum americanum foram encontrados 75 ácaros pertencentes a sete espécies, em 20 das 28 coletas.

Em Iolinidae, Pronematus anconai BAKER, (1943)

Tabela 1 - Famílias, espécie vegetal e ácaros predadores associados a plantas na cultura da videira, nos municípios de Bento Gonçalves (BG), Boqueirão do Leão (B L), Candiota (C) e Dois Lajeados (D L), no Estado do Rio Grande do Sul.

\begin{tabular}{|c|c|c|c|c|c|c|}
\hline \multirow{2}{*}{ Família / Espécie vegetal } & \multirow{2}{*}{ Ácaros predadores } & \multirow{2}{*}{$\begin{array}{l}\text { Amostragens } \\
\text { (n ácaros) }\end{array}$} & \multicolumn{4}{|c|}{ Local e $\mathrm{n}^{\circ}$ ácaros } \\
\hline & & & BG & $\mathrm{BL}$ & $\mathrm{C}$ & DL \\
\hline \multicolumn{7}{|l|}{ Amaranthaceae } \\
\hline Amaranthus deflexus L. & Neoseiulus californicus & $10(2)$ & & & 1 & \\
\hline $\begin{array}{l}\text { Amaranthus hybridus L. } \\
\text { Amaranthus sp. }\end{array}$ & & $\begin{array}{l}2 \\
9\end{array}$ & & & & \\
\hline Iresine diffusa Humb. \& Bonpl. ex Willd. & $\begin{array}{l}\text { Agistemus sp. } \\
\text { Pronematus anconai }\end{array}$ & $4(3)$ & & & & $\begin{array}{c}1 \\
14\end{array}$ \\
\hline $\begin{array}{l}\text { Apiaceae } \\
\text { Bowlesia incana Ruiz \& Pav. } \\
\text { Conium maculatum L. } \\
\text { Asteraceae }\end{array}$ & $\begin{array}{l}\text { Amblyseius vitis } \\
\text { Neoseiulus californicus }\end{array}$ & $12^{1}(2)$ & 3 & & & 1 \\
\hline & Neoseiulus californicus & & & & 1 & \\
\hline Artemisia sp. & Typhlodromalus aripo & 1 & & & 1 & \\
\hline Baccharis trimera (Less.) DC. & $\begin{array}{l}\text { Neoseiulus californicus } \\
\text { Agistemus floridanus }\end{array}$ & $11(1)$ & & & $\begin{array}{l}1 \\
1\end{array}$ & \\
\hline Bidens pilosa $\mathrm{L}$. & $\begin{array}{l}\text { Neoseiulus californicus } \\
\text { Pronematus anconai } \\
\text { Typhlodromus rhenanus } \\
\text { Zetzellia malvinae }\end{array}$ & $19(9)$ & 1 & 1 & $\begin{array}{l}1 \\
1 \\
2 \\
1\end{array}$ & 5 \\
\hline Blainvillea sp. & $\begin{array}{l}\text { Arrenoseius gaucho } \\
\text { Neoseiulus californicus }\end{array}$ & 1 & & & $\begin{array}{l}1 \\
1\end{array}$ & \\
\hline Brachiaria sp. & $\begin{array}{l}\text { Neoseiulus californicus } \\
\text { Pronematus anconai }\end{array}$ & $7(1)$ & $\begin{array}{l}2 \\
8\end{array}$ & & & \\
\hline
\end{tabular}


Tabela 1 - Continuação.

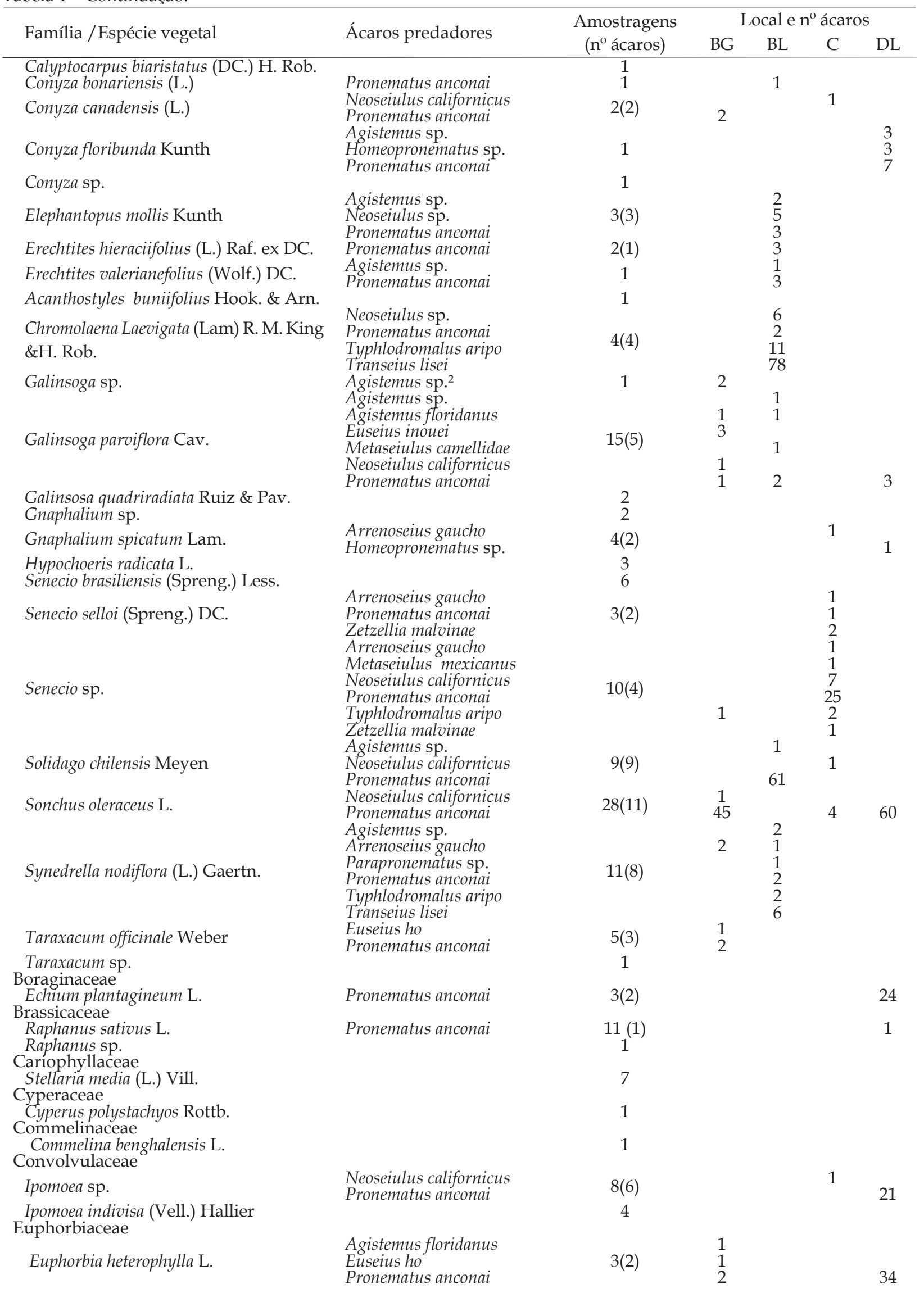

Continua... 
Tabela 1 - Continuação.

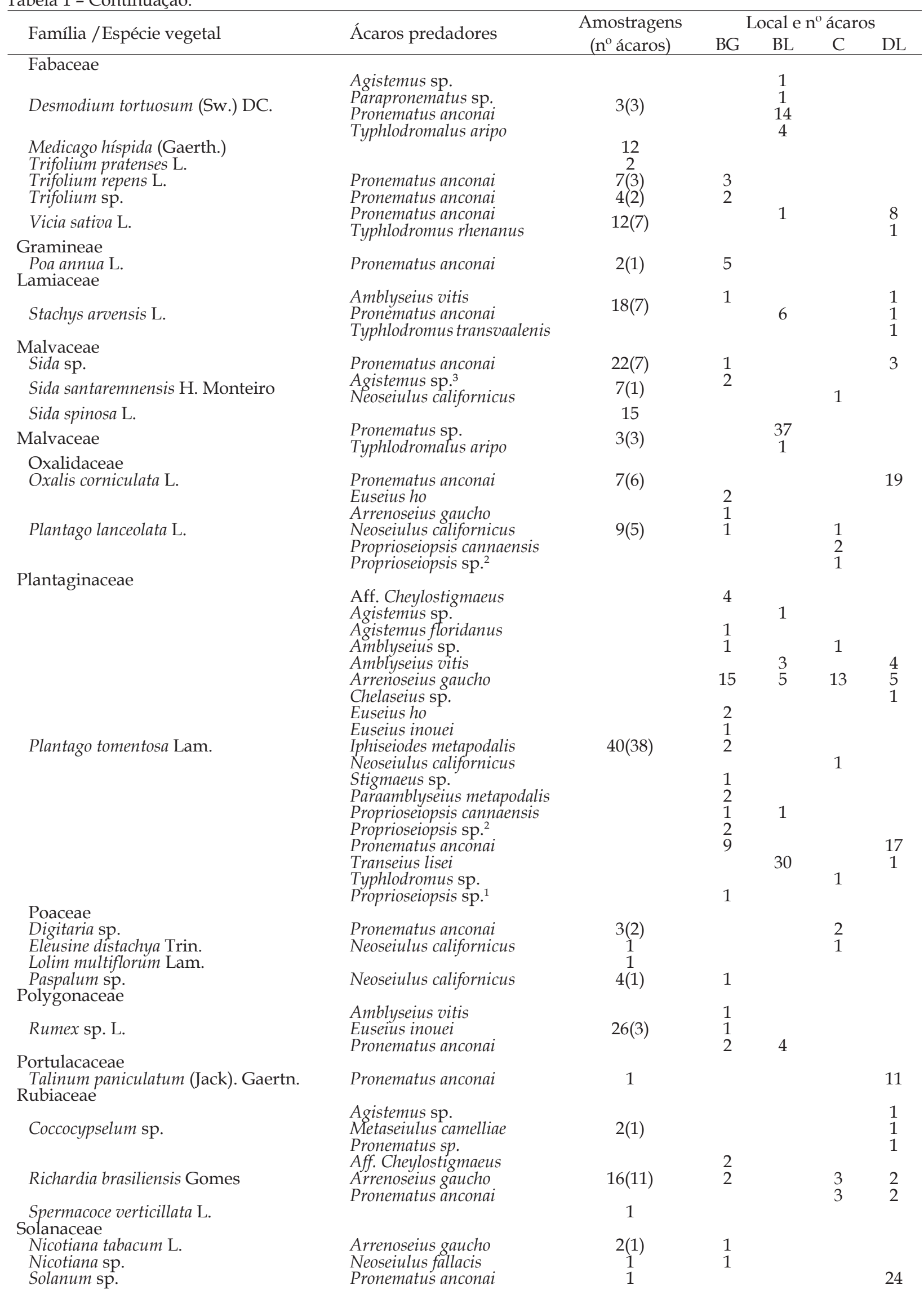

Continua... 
Tabela 1 - Continuação.

\begin{tabular}{|c|c|c|c|c|c|c|}
\hline \multirow{2}{*}{ Família /Espécie vegetal } & \multirow{2}{*}{ Ácaros predadores } & \multirow{2}{*}{$\begin{array}{c}\text { Amostragens } \\
\text { (nº ácaros) }\end{array}$} & \multicolumn{4}{|c|}{ Local e $\mathrm{n}^{\circ}$ ácaros } \\
\hline & & & BG & BL & $\mathrm{C}$ & DL \\
\hline \multirow{5}{*}{ Solanum americanum Mill. } & Agistemus sp. & & 2 & 1 & \multirow[b]{3}{*}{3} & 3 \\
\hline & Metaseiulus mexicanus & & 2 & 1 & & 1 \\
\hline & $\begin{array}{l}\text { Neoseiulus californicus } \\
\text { Neoseiulus tunus }\end{array}$ & $28(20)$ & & 4 & & 1 \\
\hline & Pronematus anconai & & & 11 & 1 & 35 \\
\hline & Typhlodromalus aripo & & & 4 & & J \\
\hline Solanum diflorum Vell. & Pronematus anconai & 1 & & 5 & & \\
\hline Solanum mauritianum Scop. & $\begin{array}{l}\text { Arrenoseius gaucho } \\
\text { Pronematus anconai }\end{array}$ & $3(1)$ & $\begin{array}{l}2 \\
4\end{array}$ & & & \\
\hline Sterculiaceae & & & & & & \\
\hline Waltheria indica L. & $\begin{array}{l}\text { Typhlodromalus aripo } \\
\text { Transeius lisei }\end{array}$ & 1 & & $\begin{array}{c}17 \\
2\end{array}$ & & \\
\hline Thelyteridaceae & A gistemus sn & & & 1 & & \\
\hline Thelypteris dentate (Forssk.) EP St. John & $\begin{array}{l}\text { Pronematus anconai } \\
\text { Typhlodromalus aripo }\end{array}$ & $8(5)$ & & $\begin{array}{c}1 \\
54 \\
7\end{array}$ & & \\
\hline & Arrenoseius gaucho & 1 & & & 1 & \\
\hline P2 & Homeopronematus sp. & 1 & & 14 & & \\
\hline P3 & $\begin{array}{l}\text { Pronematus anconai } \\
\text { Pronematus anconai }\end{array}$ & $2(2)$ & & $\begin{array}{l}6 \\
7\end{array}$ & 1 & \\
\hline P5 & Pronematus anconai & & & 7 & & \\
\hline Total geral & & & & & & \\
\hline
\end{tabular}

1944 foi mais abundante com 618 espécimes presentes em 44 plantas amostradas. Pronematus sp. com 37 indivíduos em somente uma amostral de Malvaceae. Nos municípios de Bento Gonçalves e Candiota Pronematus sp. foi encontrado associado comumente à cultura da videira (JoHANn et al., 2009; KLOCK et al., 2011). As espécies mais abundantes em Phytoseiidae foram Transeius lisei Ferla \& Silva 2008, com 112 indivíduos encontrados e em Chromolaena Laevigata, Synedrella nodiflora, Plantago tomentosa. e Waltheria indica. Typhlodromalus aripo DeLeon, 1967, com 50 espécimes e esteve presente em nove espécies amostradas, Arrenoseius gaucho (FERLA et al., 2010, com 68 indivíduos em 12 espécies distintas.

Stigmaeidae apresentou baixas populações. Foi representado por Agistemus sp. encontrado em 12 das 80 espécies amostradas, com 19 indivíduos. Baixas populações também foram encontradas por MAtioli et al. (2002) em estudo realizado em citros.

Não foram encontrados ácaros predadores em 22 espécies vegetais, bem como um baixo número em outras. O que pode ter influenciado estes resultados foi o número de vezes que estas plantas foram amostradas, demonstrando a necessidade de mais estudos com estas.

Plantago tomentosa, Sonchus oleraceus e Chromolaena Laevigata foram as plantas que apresentaram maior riqueza de espécies acarinas, o que pode estar relacionado com as características morfofisio-ógicas como a presença de pilosidades, tricomas e nervuras ao longo da folha bem destacadas que podem servir de abrigo aos predadores. Como se tratam de espécies que florescem o ano todo (LorENZI, 2000), podem fornecer pólen constantemente para o ácaro predador. Resultados semelhantes obtidos por CoLliER et al. (2001) em Malus domestica (Borkham) mostram que plantas associadas podem servir como fonte de alimento para o ácaro predador, oferecendo pólen ou hospedando presas secundárias, em época desfavorável à presa principal.

As espécies associadas à videira demonstram um papel importante como abrigo de inimigos naturais, oferecendo requisitos importantes a eles, tais como hospedeiro e presa alternativos, pólen ou néctar, bem como micro-hábitat que não disponíveis em monoculturas livre de plantas associadas (VAN EMDEN, 1965).

As quatro espécies mais abundantes de predadores, Pronematus anconai, Transeius lisei, Arrenoseius gaucho e Typhlodromalus aripo, apresentam potencial como inimigos naturais de ácaros fitófagos. Mas, torna-se necessário desenvolver mais estudos no sentido de definir sua importância nos agroecossistemas e nas espécies vegetais. Quais as perspectivas para o incremento da população de ácaros predadores importante para a manutenção dos níveis populacionais dos ácaros pragas abaixo dos níveis de dano.

Pode-se concluir que há um grande número de ácaros predadores presentes em espécies de plantas associados à videira, surgindo perspectivas para o uso destas em programas de controle biológico conservativo de ácaros-praga. 


\section{AGRADECIMENTOS}

Os autores agradecem ao Centro Universitário UNIVATES, aos bolsistas do Laboratório de Acarologia do Centro UniversitárioUNIVATES pela ajuda na identificação das espécies acarinas, à bióloga Crisna Letícia Klock pela cedência dos dados e a Fernanda Majolo pela colaboração nas traduções

\section{REFERÊNCIAS}

AGRAWAL, A.A.; R. KARBAN, COLFER, R. How leaf domatia and induced plant resistance affect herbivores, natural enemies and plant performance. Oikos, v.89, p.70-80, 2000.

ALTIERI, M.A.; SCHOONHOVEN A, V.; DOLL, J.D. The ecological role of weeds in insect pest management systems: A review with bean (Phaseolus vulgaris L.) cropping systems. PANS, v.23, p.195-205, 1977.

ALTIERI, M.A.; SILVA E.N.; NICHOLLS, C.I. O papel da biodiversidade no maneio de pragas. Ribeirão Preto: Holos, 2003. 226p.

COLLIER, K.F.S.; ALBUQUERQUE, G.S.; EIRAS, A.E.; BLACKMER, J.L.; ARAÚJO, M.C., MONTEIRO, L.B. Estímulos Olfativos Envolvidos na Localização de Presas pelo Ácaro Predador Neoseiulus californicus (McGregor) (Acari: Phytoseiidae) em Macieiras e Plantas Hospedeiras Alternativas. Neotropical Entomology, v.30, n.4, 2001.

DUSO, C.; LILLO, D.C.M. Grape. In: LINDQUIST, E.E.; SABELIS, M.W.; BRUIN, J. (Eds). Eriophyoid mites. Amsterdan: Elsevier, 1996. p.571-582.

FERLA, N.J.; MORAES, G.J. de Ácaros predadores (Acari) em plantas nativas e cultivadas do Estado do Rio Grande do Sul, Brasil. Revista Brasileira de Zoologia, Curitiba, v.19, n.4, p.1011-1031, 2002.

FERLA, N.J.; SILVA, G.L. da; MORAES, G.J. de Description of a new species of Arrenoseius wainstein (Acari: Phytoseiidae) from Brazil and a redescription of a similar species from Argentina. International Journal of Acarology, v.36, n.1, p.15-19, 2010.

JEPPSON, L.R.; KEIFER, H.H.; BAKER, E.W. Mites injurious to economic plants. Berkeley: University of California Press, 1975. 612p.

JOHANN, L.; KLOCK C.L.; FERLA, N.J.; BOTTON, M. Acarofauna (Acari) associada à videira (Vitis vinifera L.) no Estado do Rio Grande do Sul. Biociências, Porto Alegre, v.17, n.1, p.1-19, 2009.

KLOCK, C.L.; JOHANN, L.; BOTTON, M; LIMA, L.F.; FERLA, N.J. Mitefauna (Arachnida: Acari) associated to grapevine, Vitis vinifera L. (Vitaceae), in the municipalities of Bento Gonçalves and Candiota, Rio Grande do Sul, Brazil. Check List, v.7, n.4, p.522-536, 2011.

LAING, J.E.; KNOP, N.F. Potential use of predaceous mites other than Phytoseiidae for biological control of orchards pests. In:HOY, M.A.; CUNNINGHAM, G.L.; KNUTSON, L. (Ed.). Biological control of pest by mites. Berkeley: University of California Press, 1982. p.28-35.

LORENZI, H. Plantas daninhas do Brasil. 3. ed. Nova Odessa, SP: Instituto Plantarum, 2000.

MATIOLI, A.L.; UECKERMANN, E.A.; OLIVEIRA, C.A.L. Some Stigmaeid and eupalopsellid mites from citrus orchads in Brazil (Acari: Stigmaeidae and Eupalopsellidae). International Journal of Acarology, v.28, n.2, p.99-120, 2002.

MATOS, C.H.C.; PALLINI, A.; BELLINI, L.L.; FREITAS, R.C.P. de Domácias e seu papel na defesa das plantas. Ciencia Rural, v. 36, n.3, 2006.

McMURTRY, J.A.; CROFT, A. B. Life-styles of Phytoseiidae mites and their roles in biological control. Annual. Review of Entomology, v.42, p.291-321, 1997.

McMURTRY, J.A.; HUFFAKER, C.B.; VAN DEN VRIE, M.V. Ecology of tetranychid mites and their natural enemies: A review. I. Tetranychid enemies: their biological characters and the impact of spray. Hilgardia, v.40, n.11, p.331-390, 1970.

MEYER, G. de A. Flutuação populacional de Panonychus ulmi (Koch, 1836) (Acari: Tetranychidae) e seus predadores em pomares de macieira nos sistemas de produção convencional e teste de seletividade em laboratório com Neoseiulus californicus (McGregor 1954) (Acari: Phytoseiidae). 2003. 74f. Dissertação (Mestrado) - Universidade Federal de Pelotas, 2003.

MORAES, G. J. de Controle biológico dos ácaros fitófagos. Informe Agropecuário, v.5, p.55-62, 1991.

MORAES, G.J. de; McMURTRY, J.A.; DENMARK, H.A.; CAMPOS, C.B. A revised catalog of the mite family Phytoseiidae. Zootaxa, v.434, p.1-494, 2004.

MORAES, G.J. de; ZACARIAS, M.S.; GONDIM JÚNIOR, M.G.C.; FERES, R.J.F. Papel da vegetação natural como reservatório de ácaros predadores. In: SIMPÓSIO DE CONTROLE BIOLÓGICO (SICONBIOL), 7., 2001, Poços de Caldas. Anais. Poços de Caldas: 2001. p.492-497.

PARRA, J.R.P.; BOTELHO, P.S.M.; CORREA-FERREIRA, B.S. Controle biologico no Brasil: parasitoides e predadores. São Paulo: Manole, 2002.

PERRIN, T.M.; McMURTRY, J.A. Other predatory arthropods. In: LINDQUIST, E.E.; SABELIS, M.W.; BRUIN, J. (Ed.). Eriophyoid mites: their biology, natural enemies and control. Amsterdan: Elsevier, 1996. p.471-479. 
PRISCHMANN, D.A.; JAMES, D.G. Phytoseiid (Acari) on unsprayed vegetation in southcentral Washington: Implications for biological control or spider mites on wine grapes. International Journal of Acarology, v.29, p.279-287. 2003.

TIXIER, M.S.; KREITER, S.; AUGER, P. Colonization of vineyards by phytoseiid mites: their dispersal patterns in the plot and their fate. Experimental \& Applied Acarology, v.24, p.191-211, 2000.
VAN EMDEN, H.F. The role of uncultivated land in the biology of crop pests and beneficial insects. Scientific Horticulture, v.17, p.121-136, 1965.

WALTER, D.E. Living on leaves: Mites, tomenta, and leaf domatia. Annual Review of Entomology, v.8, p.307344, 1996.

Recebido em 20/12/11

Aceito em 19/10/12 